\title{
ON KADISON'S CONDITION FOR EXTREME POINTS OF THE UNIT BALL IN A $B^{*}$-ALGEBRA
}

\author{
by BERTRAM YOOD ${ }^{1}$
}

(Received 4th July 1968)

\section{Introduction}

Let $B$ be a complex Banach algebra with an identity 1 and an involution $x \rightarrow x^{*}$. Kadison (1) has shown that, if $B$ is a $B^{*}$-algebra, , the set of extreme points of its unit ball coincides with the set $\mathbb{E}$ of elements $x$ of $B$ for which

$$
\left(1-x^{*} x\right) B\left(1-x x^{*}\right)=(0) \text {. }
$$

This elegant result is very useful for $B^{*}$-algebra theory; see (1) and (3). In this paper we examine the set $\mathbb{E}$ for algebras $B$ which are not necessarily $B^{*}$-algebras. It is shown that the spectral radius of each $x \in \mathbb{E}$ is at least one. In Section 4 we consider the set $\mathfrak{E}$ for the special case where $B$ is the algebra of all bounded linear operators on the infinite-dimensional Hilbert space $H$. Here $\mathbb{E}$ is the set of semi-unitary elements $T\left(T T^{*}=1\right.$ or $\left.T^{*} T=1\right)$. For such $T$ we show that there exists a complex number $b,|b|=1$, such that $b-T$ is not a se mi-Fredholm operator on $H$. (For this notion see Section 4 or (2)). This then says that $b$ lies in the essential spectrum of $T$ when we use the rather restrictive definition of essential spectrum due to Kato (2, p. 243).

\section{Algebraic considerations}

We begin with some pure ring theory. Let $A$ be a ring with identity 1 and an involution $x \rightarrow x^{*}$. Let $\mathbb{E}(A)$ denote the set $x \in A$ for which

$$
\left(1-x^{*} x\right) A\left(1-x x^{*}\right)=(0) \text {. }
$$

For $x \in \mathbb{E}(A)$ we have $1=x x^{*} \circ x^{*} x=x^{*} x \circ x x^{*}$ where we use the familiar notation (4) that $u \circ v=u+v-u v$.

Proposition 1. Let $x \in \mathfrak{E}(A)$. Then $x^{n} \in \mathbb{E}(A)$ for $n=1,2, \ldots$.

Proof. Let $x \in \mathbb{E}(A)$. The following computations use ideas of Miles (3, p. 631).

First we show that

$$
\left(1-\left(x^{*}\right)^{n} x^{n}\right) A\left(1-x x^{*}\right)=(0)
$$

for $n=1,2, \ldots$. By hypothesis, this is valid for $n=1$ and we suppose it is true for the integer $n$. Note that

$$
1-\left(x^{*}\right)^{n+1} x^{n+1}=1-\left(x^{*}\right)^{n} x^{n}+\left(x^{*}\right)^{n}\left(1-x^{*} x\right) x^{n} .
$$

1 This research was supported by the National Science Foundation Grant NSF-GP-7516. The author is grateful to the referee for his helpful comments. 
Set $w=\left(1-\left(x^{*}\right)^{n+1} x^{n+1}\right) y\left(1-x x^{*}\right)$. Using (3), we write $w=a+b$ where

$$
\begin{aligned}
& a=\left(1-\left(x^{*}\right)^{n} x^{n}\right) y\left(1-x x^{*}\right)=0, \\
& b=\left(x^{*}\right)^{n}\left(1-x^{*} x\right) x^{n} y\left(1-x x^{*}\right)=0
\end{aligned}
$$

since $x \in \mathfrak{E}(A)$. Likewise the relation

$$
1-x^{n+1}\left(x^{*}\right)^{n+1}=1-x^{n}\left(x^{*}\right)^{n}+x^{n}\left(1-x x^{*}\right)\left(x^{*}\right)^{n}
$$

leads to the conclusion that

for $n=1,2, \ldots$

$$
\left(1-x^{*} x\right) A\left(1-x^{n}\left(x^{*}\right)^{n}\right)=(0)
$$

Now we show that $x^{n} \in \mathcal{E}(A)$ by induction. Suppose this holds for exponents $k=1, \ldots, n$. Set

$$
w=\left(1-\left(x^{*}\right)^{n+1} x^{n+1}\right) y\left(1-x^{n+1}\left(x^{*}\right)^{n+1}\right) .
$$

Using (3) and (4), we rewrite $w$ as the sum of four terms each of which must be zero by (2) and (5) and the induction hypothesis. This establishes the desired result. In particular, $x \in \mathfrak{E}(A)$ is never a nilpotent element of $A$.

For further results we assume that the involution is proper $\left(x^{*} x=0\right.$ implies $x=0$ ). One then readily verifies that the four statements $(a) x^{*} x$ is an idempotent, (b) $x x^{*}$ is an idempotent, (c) $x=x x^{*} x,(d) x^{*}=x^{*} x x^{*}$ are equivalent. We then call $x$ a partial isometry. Arguments of Miles (3, p. 630) show that any $x \in \mathbb{E}(A)$ is a partial isometry. These results can fail if the involution is not proper. For an example let $A$ be the ring of all numbers of the form $a+b i, i^{2}=-1$, under the usual operations, where $a$ and $b$ lie in the ring of integers modulo 16. For $x=a+b i$, set $x^{*}=a-b i$. Then $x=2+i$ lies in $\mathfrak{E}(A)$ but $x x^{*}$ is not an idempotent.

Proposition 2. Suppose that the involution in $A$ is proper. If $x^{n} \in \mathbb{E}(A)$ for some integer $n$ and $x$ is a partial isometry, then $x \in \mathfrak{E}(A)$.

Proof. Suppose that $x^{n} \in \mathfrak{E}(A)$ and $x$ is a partial isometry. For each $y \in A$ we can, using (1) with $x^{n}$ instead of $x$, obtain an expression for $y$ as

$$
y=\left(x^{*}\right)^{n} x^{n} y+y x^{n}\left(x^{*}\right)^{n}-\left(x^{*}\right)^{n} x^{n} y x^{n}\left(x^{*}\right)^{n} .
$$

We use (6) in $\left(1-x^{*} x\right) y\left(1-x x^{*}\right)$ and the facts $x=x x^{*} x, x^{*}=x^{*} x x^{*}$ to see that $\left(1-x^{*} x\right) y\left(1-x x^{*}\right)=0$.

We use the following language customary in the theory of von Neumann algebras. A projection is a self-adjoint idempotent. A projection $p$ is called abelian if $p A p$ is an abelian ring and pis called minimal if $p A$ is a minimal right ideal.

Proposition 3. Suppose that the involution in $A$ is proper. Let $x \in A$ be $a$ partial isometry. Then $x^{*} x$ is an abelian (minimal) projection if and only if $x x^{*}$ is an abelian (minimal) projection.

Proof. Let $p=x^{*} x, q=x x^{*}$. Suppose that $p A p$ is commutative and let $y, z \in A$. Then

$$
p x^{*} y x p x^{*} z x p=p x^{*} z x p x^{*} y x p \text {. }
$$


But $p x^{*}=x^{*} x x^{*}=x^{*}$ and $x p x^{*}=q$. Multiplying (7) on the left by $x$ and on the right by $x^{*}$ shows that $q y q z q=q z q y q$ or $q$ is an abelian projection.

Suppose that $p$ is a minimal projection. By algebraic theory, either $x p A=(0)$ or $x p A$ is a minimal right ideal. But $x p x^{*}=q \neq 0$. Then

$$
(0) \neq q A \subset x p A \text {. }
$$

Proposition 4. Suppose that the involution in $A$ is proper. Let $x \in \mathbb{E}(A)$. Suppose that $x^{*} x$ is an abelian (minimal) projection. Then $A$ is a commutative ring (division ring). Then

Proof. Let $p=x^{*} x, q=x x^{*}$. Suppose that $p$ is an abelian projection.

$$
p q p x p=p x p q p \text {. }
$$

But $x p=x=q x$. Moreover, since $x \in \mathbb{E}(A)$, we have, as noted earlier, $p \circ q=q \circ p=1$. In particular, $p q=q p$. Then

$$
p q p x p=q p x p=q p x=p q x=p x \text {. }
$$

On the other hand, $p x p q p=p x p q=p x q$. Then (8) yields $p x(1-q)=0$. We combine this with $(1-p) x(1-q)=0$ to obtain $x(1-q)=0$. This gives $p(1-q)=x^{*} x(1-q)=0$. Since $p+q-p q=1$ we get $q=1$. But, by Proposition 3, $q$ is an abelian projection. Hence $A$ is abelian.

Suppose now that $p$ is a minimal projection. First we show that $p q=0$ is impossible. For suppose otherwise. Then $p+q=1$ and $x(p+q)=x$. Consequently, $x q=0=x^{2}\left(x^{*}\right)^{2}$. Since the involution is proper, $x^{2}=0$. This contradicts Proposition 1. This implies that $p q$ is a non-zero projection. Since $p q A=p A$, we may invoke a lemma of Rickart $(4$, p. 261) to see that $p=p q$. Via Proposition 3 we also get $q=q p$. Then $p=q=1$. Since 1 is a minimal projection, $A$ is a division ring.

\section{The set $\mathcal{E}$ for a Banach algebra $B$}

For $x \in B$, a Banach algebra, we use the notation of $(4), v(x)=\lim \left\|x^{n}\right\|^{1 / n}$ where $v(x)$ is also the spectral radius of $x$. We assume that $B$ has an identity 1 and an involution $x \rightarrow x^{*}$ but do not suppose that the involution is proper.

Theorem 1. For each $x \in \mathbb{E}$ we have $v(x) \geqq 1$. If $B$ is a $B^{*}$-algebra, then $v(x)=1$ for $x \in \mathbb{E}$. that

Proof. Let $x \in \mathfrak{E}$. Proposition 1 gives $x^{n} \in \mathfrak{E}, n=1,2,3, \ldots$ This implies

$$
\left(1-\left(x^{n}\right)^{*} x^{n}\right)\left(1-x^{n}\left(x^{n}\right)^{*}\right)=0 .
$$

Hence, for $n=1,2,3, \ldots, 1$ belongs to the spectrum of $\left(x^{n}\right)^{*} x^{n}$ or $x^{n}\left(x^{n}\right)^{*}$, leading to the conclusion that

$$
\begin{aligned}
1 & \leqq v\left(\left(x^{n}\right)^{*} x^{n}\right)=v\left(x^{n}\left(x^{n}\right)^{*}\right) \\
& \leqq\left\|\left(x^{n}\right)^{*} x^{n}\right\| \leqq\left\|\left(x^{*}\right)^{n}\right\|\left\|x^{n}\right\|, \quad(n=1,2,3, \ldots) .
\end{aligned}
$$

Therefore $1 \leqq v\left(x^{*}\right) v(x)=v(x)^{2}$. 
If $B$ is a $B^{*}$-algebra, Proposition 1 and the cited result of Kadison (1) make $\left\|x^{n}\right\|=1$, for $x \in \mathbb{E}, n=1,2,3, \ldots$ Hence $v(x)=1$.

We show, by example, that one can have a Banach algebra $B$ where $v(x)$ is as large as desired for a suitable $x \in \mathcal{E}$. Let $n$ be a positive integer, and let $Y$ be the subset of the real line, $Y=[0,1] \cup\{2,3\}$ with the usual topology. Let $C(Y)$ be the Banach algebra of all complex continuous functions on $Y$ with the sup norm. We define an involution $x \rightarrow x^{\#}$ on $Y$ by the rule that $x^{\#}(t)=\overline{x(t)}$ if $t \in[0,1], x^{\#}(2)=\overline{x(3)}$ and $x^{\#}(3)=\overline{x(2)}$. One sees that the function $x(t)=1, t \in[0,1], x(2)=n, x(3)=n^{-1}$ lies in $\mathbb{E}$ and $v(x)=n$.

Corollary 1. Let $K$ be a proper two-sided closed *-ideal of $B$. Then

$$
\text { dist }(\mathfrak{F}, K) \geqq 1 \text {. }
$$

Proof. Let $\pi$ be the natural homomorphism of $B$ onto $B / K$ and let $x \in \mathbb{E}$. Clearly $\pi(x) \in \mathcal{E}(B / K)$ and, by Theorem 1 , dist $(x, K)=\|\pi(x)\| \geqq v(\pi(x)) \geqq 1$.

Corollary 2. Let $B_{1}$ be $a B^{*}$-algebra with an identity and $T$ be an algebraic *-homomorphism of $B_{1}$ onto a dense subset of $B$. Then $v(T(x))=1$ and $\|T(x)\| \geqq\|x\|$ for each $x \in \mathbb{E}\left(B_{1}\right)$.

Proof. In this situation, $T(x) \in \mathfrak{E}(B)$. Then, by Theorem 1 ,

$$
v(x) \geqq v(T(x)) \geqq 1=v(x)=\|x\| .
$$

Since we also have $\|T(x)\| \geqq v(T(x))$, the desired relations follow.

\section{The set $\mathcal{E}$ for operator algebras}

First we consider the algebra $B(X)$ of all bounded linear operators on a complex Banach space $X$ and the closed two-sided ideal $K(X)$ of compact operators. Let $R(T)$ denote the range of $T \in B(X)$. We define nul $(T)$ as the dimension of $T^{-1}(0)$ and def $(T)$ as the dimension of $X / R(T)$ (these are called $\infty$ if they are not finite). As usual (2) $T$ is called semi-Fredholm if $R(T)$ is closed and either nul $(T)<\infty$ or def $(T)<\infty$. If $R(T)$ is closed and both nul $(T)<\infty$, $\operatorname{def}(T)<\infty, T$ is said to be a Fredholm operator. For a Fredholm operator we take as its index, ind $(T)=\operatorname{nul}(T)-\operatorname{def}(T)$.

Let $\sigma$ denote the natural homomorphism of $B(X)$ onto $B(X) / K(X)$. Following (2), p. 242, we let $\Delta=\Delta(T)$ denote the semi-Fredholm region for $T \in B(X)$. This is the set of complex numbers $a$ for which $a-T$ is a semi-Fredholm operator. Also $\Delta_{F}$ denotes the subset consisting of all $a$ for which $a-T$ is a Fredholm operator. Then $\Delta_{F}$ can also be described as the $a$ for which $\sigma(a-T)$ has a two-sided inverse in $B(X) / K(X)$; see (5), p. 617. We are also concerned with the essential spectrum $\Sigma_{e}=\Sigma_{e}(T)$ in the sense of (2), p. 243, which is the complement of $\Delta(T)$. That $\Delta_{F} \cup \Sigma_{e}$ does not in general exhaust the complex plane adds interest to Theorem 2 . We use the notation

$$
r=r(T)=\lim \left\|(\sigma(T))^{n}\right\|^{1 / n} \text {. }
$$

Theorem 2. Let $T \in B(X)$ where $X$ is infinite-dimensional. Then each complex number $a,|a|=r$, lies in $\Delta_{F} \cup \Sigma_{e}$. At least one such a lies in $\Sigma_{e}$. 
Proof. Let $|a|=r$ and suppose that $a \notin \Delta_{F}$. First-we show that $a-T$ cannot have a closed range together with nul $(a-T)<\infty$. For suppose otherwise. As $a \notin \Delta_{F}$, def $(a-T)=\infty$. Let $x_{1}, \ldots, x_{n}$ be a (finite) basis for the null space of $a-T$. There exist $y_{1}, \ldots, y_{n}$ in $X$, linearly independent modulo $R(a-T)$. Choose $x_{j}^{*} \in X^{*}, j=1, \ldots, n$, such that $x_{j}^{*}\left(x_{k}\right)=\delta_{j k}, j, k=1, \ldots, n$ and set

$$
V(x)=\sum_{j=1}^{n} x_{j}^{*}(x) y_{j}
$$

Then $V\left(x_{j}\right)=y_{j}, j=1, \ldots, n$ and $V \in K(x)$.

We claim that $a-T-V$ is one-to=one. For suppose $V(z)=(a-T)(z)$. Then $(a-T)(z)$ is a linear combination of $y_{1}, \ldots, y_{n}$ so that $(a-T)(z)=0=V(z)$. Then $z$ can be written as $z=b_{1} x_{1}+\ldots+b_{n} x_{n}$ and $V(z)=b_{1} y_{1}+\ldots+b_{n} y_{n}=0$. Thus each $b_{j}=0$ and $z=0$.

It is clear that $R(a-T-V) \subset R(a-T) \oplus R(V)$. To see the reverse set inequality, suppose that $u=(a-T)(x)$ and $v=V(y)$. Let

$$
w=\sum_{i=1}^{n} x_{i}^{*}(y) x_{i}, \quad z=x-\sum_{i=1}^{n} x_{i}^{*}(x) x_{i} .
$$

Then an easy computation shows that

$$
(a-T-V)(z-w)=u+v .
$$

Hence $R(a-T-V)$ is closed, $R(a-T-V) \neq X$. By (5), p. 618, there exists $\varepsilon>0$ such that, for $|\lambda-a|<\varepsilon, \lambda-T-V$ is a one-to-one bicontinuous linear mapping of $X$ onto a proper closed subspace of $X$. This is the case for a special choice of a complex number $b,|b|>|a|,|b-a|<\varepsilon$. Since $|b|>r$, $b-T$ is invertible in $B(X) / K(X)$ and $b-T-V$ is a Fredholm operator. In view of (5), Lemma 2.4, ind $(b-T-V)=0$. But $\operatorname{nul}(b-T-V)=0$ so that $\operatorname{def}(b-T-V)=0$. But then $R(b-T-V)=w$, which is a contradiction.

We show next that $a-T$ cannot be semi-Fredholm with nul $(a-T)=\infty$ and $\operatorname{def}(a-T)<\infty$. For suppose otherwise. Then $a-T^{*}$ is a semi-Fredholm operator on the Banach space $X^{*}$, nul $\left(a-T^{*}\right)<\infty$, def $\left(a-T^{*}\right)=\infty$, whereas $\lambda-T^{*}$ is a Fredholm operator if $|\lambda|>|a|$. The above reasoning will again lead to a contradiction.

Finally not all complex numbers $a,|a|=r$ can be in $\Delta_{F}$. For suppose otherwise. First consider the case $r=0$. Then the spectrum of $\sigma(T)$ in

$$
B(X) / K(X)
$$

would be void. For the case $r>0$, we use the fact that $\Delta_{F}$ is open. Note that $\lambda \in \Delta_{F}$ if $|\lambda|>r$. Then there exists $s<r$ such that $\lambda \in \Delta_{F}$ if $|\lambda|>s$. But then $\sigma(\lambda-T)$ is invertible in $B(X) / K(X)$ for all $|\lambda|>s$. This makes $r<s$, which is a contradiction.

Corollary 3. Let $H$ be an infinite-dimensional Hilbert space and $T$ be a semiunitary element of $B(H)$. Then there exists a complex number $b,|b|=1$, which lies in $\Sigma_{e}(T)$.

E.M.S. - $Q$ 
Proof. Consider again the natural homomorphism $\sigma$ of $B(H)$ onto

$$
B(H) / K(H) \text {. }
$$

Then $\sigma(T)$ is a semi-unitary element of the quotient algebra and $r(T)=1$ in the notation of Theorem 2. By that result, the desired conclusion follows.

\section{REFERENCES}

(1) R. V. KADISON, Isometries of operator algebras, Ann. of Math. (2) 54 (1951), 325-338.

(2) T. KATO, Perturbation Theory for Linear Operators (Springer-Verlag, 1966).

(3) P. Mres, $B^{*}$-algebra unit ball extreme points, Pacific J. Math. 14 (1964), 627-736.

(4) C. E. RickART, General Theory of Banach Algebras (Van Nostrand, 1960).

(5) B. Yoon, Difference algebras of linear transformations on a Banach space, Pacific J. Math. 4 (1954), 615-636.

UNIVERSITY OF OREGON

EUGENE, OREGon, U.S.A. 